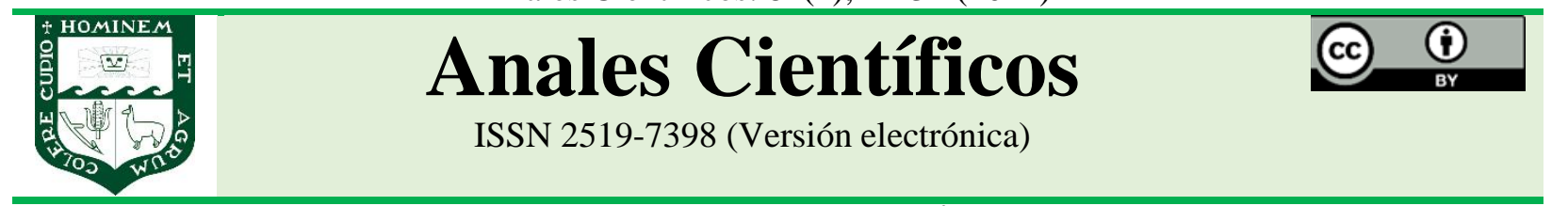

ARTÍCULO ORIGINAL - RESEARCH ARTICLE

http://dx.doi.org/10.21704/ac.v82i1.1738

\title{
USO INDISCRIMINADO DE PLAGUICIDAS EN LA CADENA DE VALOR DEL ROCOTO (Capsicum pubescens) EN OXAPAMPA
}

\section{Indiscriminated use of pesticides in the rocoto value chain (Capsicum pubescens) in Oxapampa}

\author{
Vilma E. Gómez Galarza ${ }^{1 *(1)}$ \\ ${ }^{1}$ Facultad de Economía y Planificación, Universidad Nacional Agraria La Molina, La Molina, 15024, Lima, Perú. \\ * E-mail:vgg@lamolina.edu.pe
}

Recibido: 13/11/2019; Aceptado: 02/03/2021; Publicado: 31/08/2021

\begin{abstract}
The research presents evidence of the intensive use of chemical pesticides along the links of the value chain of rocoto from the province of Oxapampa (Pasco Region) to the mercado de Lima. The value chain approach was used to analyze the production and supply of rocoto to Metropolitan Lima. The information gathering techniques were formal interviews, observation and review of current regulations on the use of pesticides. Oxapampa is the main supplier of fresh hot pepper to Metropolitan Lima. The results showed that all producers used chemical pesticides at harvest and postharvest, representing $22 \%$ of production costs. In the commercialization, the gatherers applied fungicides before sending the fresh rocoto to the Lima market. At the level of wholesalers and retailers, participation was evidenced by influencing decisions on the use of pesticides through the demands of rocoto fruits, of large size, color and without spots. The use of pesticides recommended for other crops was generalized, with prohibited active ingredients and restricted use in other countries (benomyl, paraquat and glyphosate). The practices in the management of pesticides in the links of production and marketing in the value chain of rocoto put at risk both the health of producers and consumers, the environment, and the continuity of this productive activity.
\end{abstract}

Keywords: Rocoto; Value chains; pesticides; good practices; environment

\section{RESUMEN}

La investigación presenta evidencias del uso intensivo de plaguicidas químicos a lo largo de los eslabones de la cadena de valor del rocoto desde la provincia de Oxapampa (Región Pasco) al mercado de Lima. Se utilizó el enfoque de cadena de valor para analizar la producción y el abastecimiento de rocoto a Lima Metropolitana. Las técnicas de recopilación de información fueron entrevistas formales, observación y revisión de la normatividad vigente sobre el uso de plaguicidas. Oxapampa es la principal zona proveedora de rocoto fresco a Lima Metropolitana. Los resultados mostraron que todos los productores utilizaban plaguicidas químicos en la cosecha y poscosecha, representando el $22 \%$ de los costos de producción. En la comercialización, los acopiadores aplicaron fungicidas antes de enviar el rocoto fresco al mercado de Lima. A nivel de los mayoristas y los minoristas se evidenció la participación al influir en las decisiones en el uso de plaguicidas a través de las exigencias de frutos de rocoto, de gran tamaño, color y sin manchas. Fue generalizado el uso de plaguicidas recomendados para otros cultivos, con ingredientes activos prohibidos y de uso restringido en otros países (benomyl, paraquat y glyphosate). 
Las prácticas inadecuadas en el manejo de plaguicidas en los eslabones de la producción y comercialización en la cadena de valor del rocoto ponen en riesgo tanto la salud de productores como de consumidores, del medio ambiente, y la continuidad de esta actividad productiva.

Palabras clave: Rocoto; cadenas de valor; plaguicidas; buenas prácticas; medio ambiente

Forma de citar el artículo (Formato APA):
Gomez, V. (2021). Uso indiscriminado de plaguicidas en la cadena de valor del rocoto (Capsicum pubescens) en Oxapampa.
Anales Científicos. 82(1), 22-32. http://dx.doi.org/10.21704/ac.v82i1.1738
Autor de correspondencia (*):Vilma Gómez Galarza. Email: vgg@lamolina.edu.pe
( Los autores. Publicado por la Universidad Nacional Agraria La Molina.
This is an open access article under the CC BY

\section{INTRODUCCIÓN}

El rocoto (Capsicum pubescens) es un tipo de ají oriundo del Perú que data de épocas pre-incaicas. Existe evidencia de su consumo desde 3000 años a.c. en Caral, la civilización más antigua de América (APEGA, 2009). Actualmente sus áreas cultivadas y de consumo han aumentado incentivados por el crecimiento de la gastronomía, el turismo y la comida étnica.

Oxapampa se ubica en la selva central de la región Pasco. Se encuentra a $400 \mathrm{~km}$ de la capital por vía terrestre y es la zona de producción que abastece desde hace más de dos décadas entre el $90 \%$ al $95 \%$ de los volúmenes de rocoto que se comercializan en el mercado de Lima Metropolitana (MINAG, 2013). El consumo de plaguicidas en el Perú se ha incrementado notablemente en los últimos 20 años. Según la FAO, en el año 2012 el Perú importó plaguicidas de distinta naturaleza por un valor total de \$US 171 millones a diferencia del año 1990 que se importó por un valor total de 14 millones de US\$. El rocoto es un fruto expuesto directamente a los productos químicos, en especial a los plaguicidas y como su consumo, sobre todo es en fresco, constituye un producto cuya producción y consumo presenta diferentes niveles de riesgo.

El objetivo de este estudio fue demostrar el uso de plaguicidas por los diferentes actores en los eslabones de la cadena de valor del rocoto, estructurada entre la provincia de Oxapampa y el mercado de Lima. Esto es de gran importancia porque las oportunidades de mercado a nivel internacional y nacional será cada vez más significativa por productos con buenas prácticas agrícolas y limpios de productos químicos.

El Proyecto "El desarrollo de cadenas de valor de los Capsicum para la conservación de la biodiversidad y el mejoramiento de los medios de vida rurales." permitió realizar investigaciones con trabajos de campo en diferentes zonas del país que produce capsicum.

\section{MATERIALES Y MÉTODOS}

Se utilizó el enfoque de la cadena de valor (Briz, 2011) que permitió la identificación de los diferentes agentes o eslabones de la cadena desde Oxapampa hasta el mercado de Lima. El concepto de cadena permitió evidenciar dicotomías entre competitividad y sostenibilidad en una cadena productiva (Astudillo, 2019).

La identificación de los fungicidas se realizó mediante un registro de uso en el manejo técnico por los diferentes agentes que intervienen desde la producción hasta el consumo de rocoto.

Como metodología se combinaron técnicas: entrevistas formales a productores, acopiadores y comerciantes mayoristas; observaciones directas de las prácticas y uso de plaguicidas, así como la revisión bibliográfica sobre las propiedades y características de los plaguicidas.

\section{El área de estudio}

Comprendió la provincia de Oxapampa sobre todo los distritos de Chontabamba, Huancabamba, Oxapampa y Villa Rica, como centros importantes 
de producción de rocoto, donde se ubica el $19 \%$ de las áreas cultivadas en el Perú (INEI, 2012).

Las áreas del cultivo de rocoto de la provincia de Oxapampa se ubican en el flanco oriental de la cordillera de los Andes, también denominada selva alta o ceja de selva con altitudes que van desde los 1000 a 2200 msnm. Estas características dan a la zona condiciones agroclimáticas muy favorables para la producción de rocoto.

La cercanía de la zona al mercado más importante del país (Lima Metropolitana) ha favorecido el desarrollo del rocoto y de otros cultivos con fines comerciales.

En Oxapampa se produce rocoto todo el año, los meses de mayor cosecha están entre los meses de agosto a octubre. La ciudad de Oxapampa, capital de la provincia, se encuentra a 9 horas de viaje por carretera desde la ciudad de Lima.

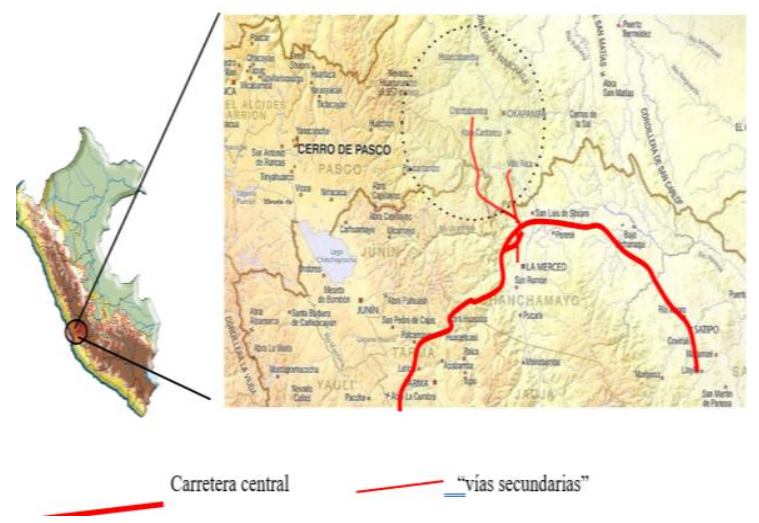

Figura 1. Oxapampa. Acceso y ubicación de la zona de Estudio (Google map, 2015).

\section{RESULTADOS Y DISCUSIÓN}

Seguidamente se presentan datos que demuestran que la demanda de rocoto ha aumentado significativamente tanto en el mercado nacional como internacional configurando el contexto para el desarrollo de una cadena de valor específica y que explica la actuación de los diferentes agentes, desde la producción hasta el consumo.

\section{Incremento de la demanda Interna e internacional del rocoto}

En el mercado interno el incremento se constató a través de las altas tasas de crecimiento en los últimos 15 años, de los volúmenes de abastecimiento de rocoto al mercado mayorista de Lima (Figura 2). Este volumen en $\mathrm{t}$ se ha incrementado notablemente de 9000 t en el año 1997 a 25000 t en el año 2013.

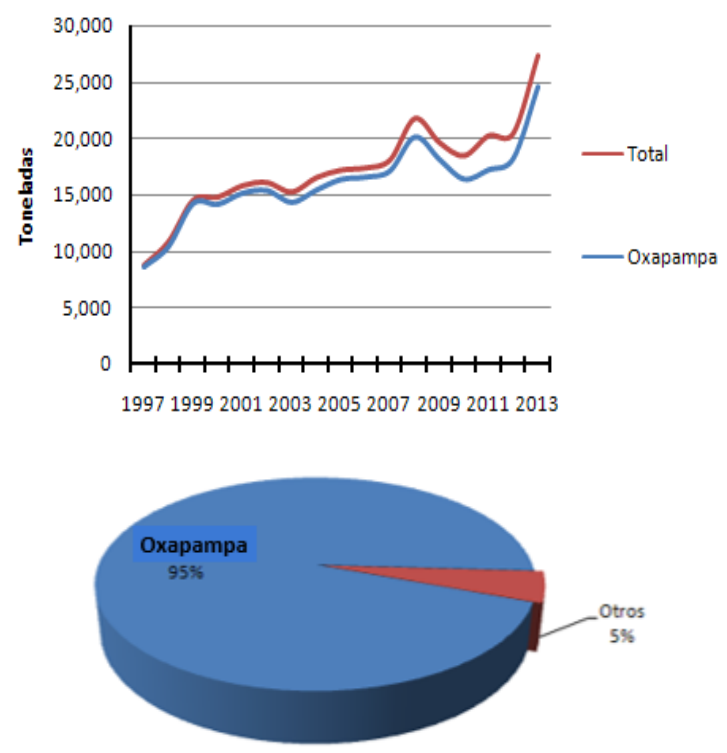

Figura 2. Evolución y participación en el abastecimiento de rocoto al mercado mayorista de Lima procedente de Oxapampa (Modificado de MINAG 2013).

La Figura 2, evidencia que, en el periodo del 19972013 la tasa de crecimiento fue de $4.4 \%$ y que el $95 \%$ del abastecimiento total de rocoto al mercado mayorista procedió de Oxapampa. La participación de otras zonas fue poco significativa.

Respecto a la demanda de los mercados internacionales, este ha crecido, sobre todo en aquellos países con poblaciones de migrantes procedentes de Perú y Ecuador. Esta dinámica se muestra a través de los volúmenes de las exportaciones del rocoto fresco y refrigerado, que ha crecido a una tasa promedio anual del 14\% (20092013) (Figura 3 y Tabla 1). 
El aumento de la demanda de rocoto ha evolucionado paralelamente con el incremento de los precios y la calidad del fruto.

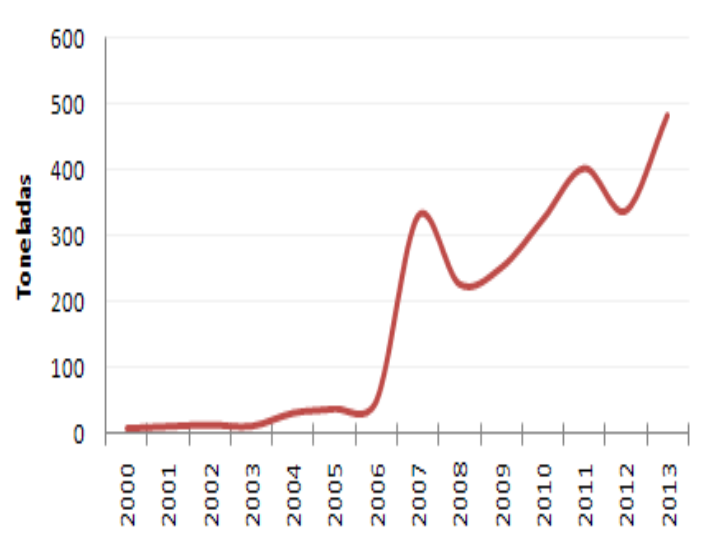

Figura 3. Perú. Exportaciones de rocoto fresco/refrigerado. 2000-2013 (SUNATADUANAS).

\section{La cadena de valor del rocoto de Oxapampa al Mercado Mayorista de Lima}

La Figura 4 muestra los diferentes eslabones que se estructuran desde Oxapampa hasta el Mercado de Lima. Por esta cadena circula entre el $90 \%$ a $95 \%$ de la producción de rocoto de Oxapampa. Se identificó, también, que se traslada rocoto en volúmenes reducidos entre el $10 \%$ al 5\%, desde Oxapampa a otros mercados como el de Huancayo, Arequipa y los mercados del Norte. Estos mercados, aunque cuentan con abastecimiento de rocoto de sus zonas circundantes, demandan los rocotos de Oxapampa por las características especiales de sus frutos, requeridos por la gastronomía regional. En la Figura 4 se reproducen los flujos de productos, dinero e información.

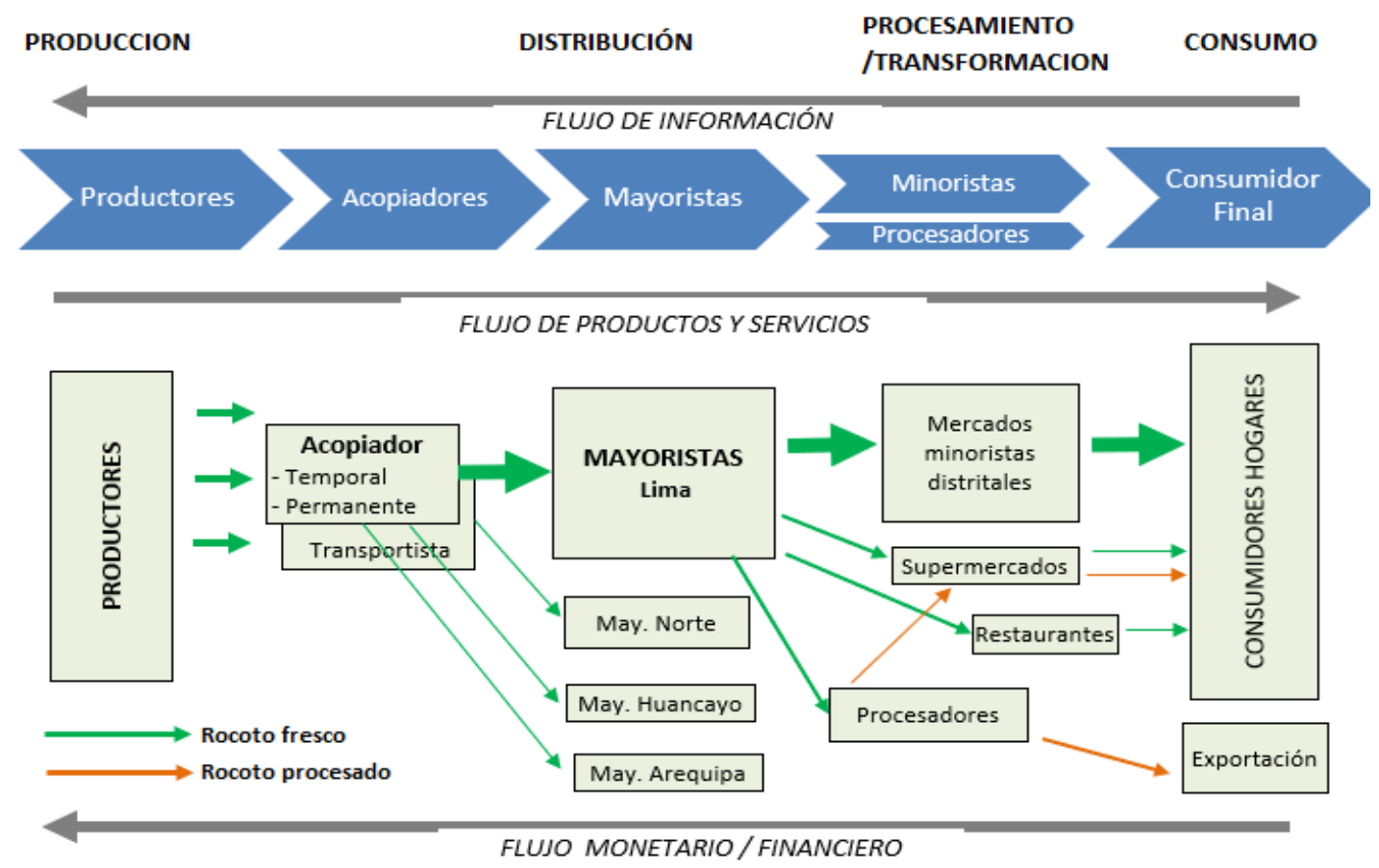

Figura 4. Estructura de la cadena de valor del rocoto de Oxapampa.

Los agentes mayoristas concentran los volúmenes de rocoto que llegan a través de los acopiadores y transportistas. De este eslabón se distribuyen a los diferentes agentes minoristas (minoristas de los mercados distritales, supermercados, restaurantes) y a los procesadores que producen productos transformados para el mercado nacional e internacional. 
Tabla 1. Perú. Exportaciones de rocoto fresco/refrigerado. 2000-2013 (SUNATADUANAS).

\begin{tabular}{ccc}
\hline Año & Toneladas & $\begin{array}{c}\text { Miles de US\$ } \\
\text { (A precios FOB) }\end{array}$ \\
\hline 2000 & 9 & 14 \\
2001 & 12 & 15 \\
2002 & 15 & 18 \\
2003 & 13 & 10 \\
2004 & 33 & 40 \\
2005 & 39 & 62 \\
2006 & 51 & 61 \\
2007 & 331 & 640 \\
2008 & 228 & 434 \\
2009 & 253 & 539 \\
2010 & 326 & 757 \\
2011 & 404 & 956 \\
2012 & 339 & 815 \\
2013 & 484 & 1.280 \\
\hline
\end{tabular}

\section{Los agentes de la cadena y el uso de agroquímicos} -El eslabón de la producción

En la zona de Oxapampa, el cultivo del rocoto con fines comerciales data de los años 70. Los productores son en su mayoría de origen alto andino: principalmente de Huánuco, Junín, Pasco, Apurímac. Son alrededor de 900 y cultivan en 800 has (INEI, 2012). En promedio, las parcelas son de 0,7 ha. Son también productores de café, granadilla, caigua, zapallo y otras hortalizas que destinan al mercado de Lima.

Para la mayoría de los productores, el rocoto constituye la "caja chica" porque les provee de liquidez por su venta semanal y/o quincenal, durante todo el año.

\section{- Tecnología productiva}

La tecnología del cultivo de rocoto es una combinación de prácticas tradicionales con el uso intensivo de agroquímicos. La Tabla 3 resume las características de las prácticas agrícolas en este cultivo. De todas estas prácticas es importante señalar que la semilla es obtenida de su propia parcela, los pocos viveros que hay en la zona son rústicos y con problemas fitosanitarios. Se cultiva rocoto en tierras con pendiente que lo obtienen quemando el bosque. Además, es un cultivo con gran demanda de mano de obra.
En esta zona, el rendimiento promedio es de $20 \mathrm{t} / \mathrm{ha}$ que duplica el alcanzado a nivel nacional (10 t/ha). Los frutos que se cosechan en estas zonas son de gran tamaño, catalogados en el mercado mayorista como extra y súper extra. Explican estos resultados las favorables condiciones agroclimáticas y el uso intensivo de agroquímicos.

Tabla 2. Características de la producción agrícola del rocoto en Oxapampa.

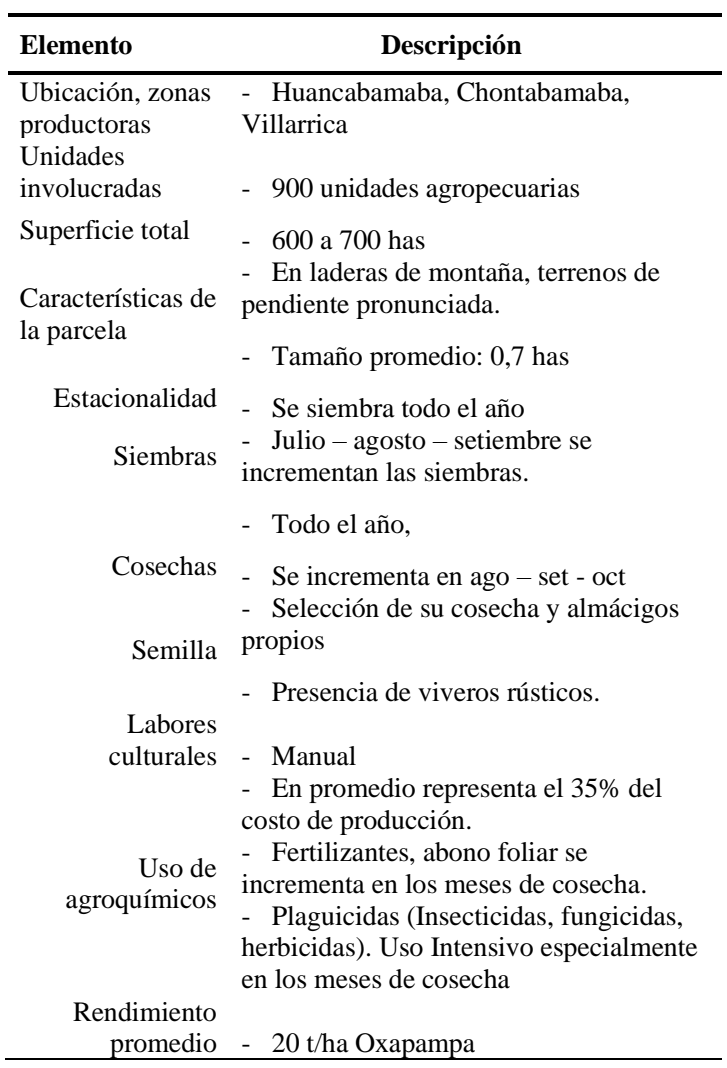

\section{- Los productores y el uso de agroquímicos}

Los agroquímicos utilizados por la totalidad de productores de rocoto son los fertilizantes, los plaguicidas (insecticidas, fungicidas, herbicidas) y abonos foliares. Durante todo el ciclo productivo del rocoto los productores manifiestan que afrontan diversos y frecuentes problemas fitosanitarios. $\mathrm{La}$ Tabla 2 muestra la frecuencia de estos problemas. Destaca la "roya", "crespadera", "chupadera y "puntos negros", con diferente percepción de su incidencia en los lugares de producción. Para el manejo de los problemas fitosanitarios todos los productores utilizan los plaguicidas químicos. 
Tabla 3. Problemas fitosanitarios mencionados por los productores de rocoto.

\begin{tabular}{lccccc}
\hline Nombre común & $\begin{array}{c}\text { Chontabamba } \\
\text { \% }\end{array}$ & $\begin{array}{c}\text { Huancabamba } \\
\text { \% }\end{array}$ & $\begin{array}{c}\text { Oxapampa } \\
\text { \% }\end{array}$ & $\begin{array}{c}\text { Villa Rica } \\
\text { \% }\end{array}$ & $\begin{array}{c}\text { Total } \\
\text { \% }\end{array}$ \\
\hline Roya & 14 & 26 & 18 & 8 & 16 \\
Crespadera & 4 & 26 & 29 & 25 & 15 \\
Chupadera & 11 & 23 & 24 & 8 & 15 \\
Punta/ puntos negros & 11 & 26 & & & 11 \\
Botritis & 7 & & 6 & 25 & 8 \\
Pela Pela & 8 & & & 25 & 8 \\
Mosca Blanca & 4 & & & 8 & 4 \\
Pulgón & 8 & & & & 4 \\
Antracnosis & 7 & & 6 & & 3 \\
Podredumbre & 7 & & & & 3 \\
Ácaros & 6 & & 6 & & 3 \\
Grillo & 6 & & 6 & & 1 \\
Herida de Tallo & 6 & & 100 & 100 & 100 \\
Nematodos & & 100 & 5 & 6 & 49 \\
Hongos & 100 & 15 & &
\end{tabular}

Los agroquímicos son adquiridos por los productores en los locales comerciales de las localidades de Oxapampa y Villa Rica cuyos vendedores aconsejan la aplicación de determinados plaguicidas. Los productores no intercambian información sobre el uso de agroquímicos y el Estado no está presente en esta zona. Los agroquímicos representan en promedio el 35\% del costo de producción y son utilizados durante todo el periodo vegetativo del cultivo de rocoto. Los plaguicidas representaron el $22 \%$ del costo total. Los rubros de gasto más importantes en agroquímicos corresponden a los fungicidas e insecticidas, lo que indica que los mayores problemas en la producción de rocoto son los hongos y los insectos. Así mismo, como la preocupación de los productores de rocoto era lograr una producción de frutos sanos y grandes, recurrieron al uso intensivo de abonos foliares que fueron aplicados sobre todo en el período de la cosecha juntamente con los plaguicidas. La aplicación de estos fue intensiva con intervalos de 15 a 20 días (Tabla 4).

Tabla 4. Distribución del gasto* en agroquímicos según la etapa del cultivo en Rocoto, Oxapampa (soles).

\begin{tabular}{|c|c|c|c|c|c|c|c|c|c|c|}
\hline \multirow{2}{*}{$\begin{array}{l}\text { Etapa del } \\
\text { cultivo }\end{array}$} & \multirow[b]{2}{*}{ Abono } & \multicolumn{2}{|l|}{ Fertilizantes } & \multicolumn{4}{|c|}{ Plaguicidas } & \multirow{2}{*}{$\begin{array}{l}\text { Aditivos } \\
\text { Adherente }\end{array}$} & \multicolumn{2}{|c|}{ TOTAL } \\
\hline & & $\begin{array}{l}\text { Fertilizante } \\
\text { sólido }\end{array}$ & Foliares & Herbicida & Fungicida & Insecticida & Acaricida & & s/. & $\%$ \\
\hline Almácigo & 25 & & & 30 & & & & & 55 & 4 \\
\hline Trasplante & & 35 & 35 & & 40 & 40 & & & 150 & 10 \\
\hline $\begin{array}{l}\text { Desarrollo } \\
\text { Vegetativo }\end{array}$ & & & & & & & 37 & & 37 & 2 \\
\hline Floración & & 35 & 70 & 30 & 80 & 80 & 37 & 50 & 382 & 25 \\
\hline Cosecha & & 70 & 175 & 60 & 200 & 200 & 111 & 75 & 891 & 59 \\
\hline ene-feb & & 35 & 35 & 30 & 40 & 40 & 37 & 25 & 242 & 16 \\
\hline mar-abr & & & 70 & & 80 & 80 & & 50 & 280 & 18 \\
\hline may-jul & & 35 & 70 & 30 & 80 & 80 & 74 & & 369 & 24 \\
\hline TOTAL & 25 & 140 & 280 & 120 & 320 & 320 & 185 & 125 & 1515 & 100 \\
\hline$\%$ & 2 & 9 & 18 & 8 & 21 & 21 & 12 & 8 & 100 & \\
\hline
\end{tabular}

(*) área de referencia $1 / 4$ de ha. 
Los plaguicidas y abonos foliares de mayor uso en la zona reportados por los productores (Tabla 5) figuran en la lista de productos autorizados por SENASA. Dentro del sistema Integrado de Gestión de Insumos Agropecuarios, (https://www.senasa.gob.pe/senasa/productos-

registrados/) se provee una lista con plaguicidas con ingredientes activos que representan serios riesgos tanto para la salud humana como para el medio ambiente. Los ingredientes activos que en otros países han sido prohibidos o aceptados con severas restricciones son: benomyl, paraquat y glyphosate.
En el caso del benomyl, la principal preocupación tiene que ver con sus efectos crónicos de distinta índole. (malformaciones congénitas severas en los ojos). Existen casos documentados en Estados Unidos y en la Unión Europea, sobre este agrotóxico que ha sido eliminado de los registros en estos países (RAP-AL, 2008). En el Perú, los distribuidores de agroquímicos recomiendan el uso del benomyl para los cultivos de algodón, espárrago, frijol, papa, vid, zapallo y en el trabajo de campo en Oxapampa se encontró que son utilizados por la mayoría de los productores en el cultivo de rocoto.

Tabla 5. Plaguicidas actualmente usados en el cultivo de Rocoto en Oxapampa.

\begin{tabular}{|c|c|c|c|c|c|}
\hline Tipo & $\begin{array}{c}\text { Nombre en la } \\
\text { zona }\end{array}$ & Nombre comercial & $\begin{array}{l}\text { Ingrediente activo } \\
\text { /composic. química }\end{array}$ & Nombre común & Registro SENASA \\
\hline \multirow[t]{4}{*}{ Fungicidas } & Benomex & Benomex & & $\begin{array}{l}\text { benomyl /benomil } \\
\text { /benomilo }\end{array}$ & 764-98-AG-SENASA \\
\hline & Benzomil & Benzomil 500 & $\begin{array}{l}\text { methyl 1-(butylcarbamoy) } \\
\text { benzimidazol-2- } \\
\text { ylcarbamate }\end{array}$ & Benomyl & $\begin{array}{l}\text { 237-96-AG-SENASA- / PQUA } \\
\mathrm{N}^{\circ} 700 \text {-SENASA }\end{array}$ \\
\hline & Sumisclex & Sumisclex 50\% PM & Procymidone & & PQUA N $^{\circ} 085$-SENASA \\
\hline & Forte & Forte 50 PM & Iprodione & & PQUA N ${ }^{\circ} 187$-SENASA \\
\hline $\begin{array}{l}\text { Insecticida / } \\
\text { acaricida }\end{array}$ & Abamex & Abamex & Abamectin & & 457-97-AG-SENASA \\
\hline Insecticidas & Lannate & $\begin{array}{l}\text { Lannate } 40 \mathrm{SO} / \\
\text { Lannate } 90 \\
\text { /Lannate LV }\end{array}$ & Methomyl & metomil / & $\begin{array}{l}\text { 767-98-AG-SENASA / 201-96- } \\
\text { AG-SENASA / 745-98-AG- } \\
\text { SENASA }\end{array}$ \\
\hline $\begin{array}{l}\text { Nematicida } \\
\text { /insecticida }\end{array}$ & Vydate & Vydate L & Oxamyl & & 195-96-AG-SENASA \\
\hline \multirow[t]{3}{*}{ Insecticida } & Cipermex & $\begin{array}{l}\text { Cipermexsuper } 10 \\
\text { CE }\end{array}$ & alpha-cypermethrin & Alfacipermetrina & 428-97-AG-SENASA \\
\hline & Meticarb & METIOCARB 90 & Methomyl & Metomil & 440-97-AG-SENASA \\
\hline & & $\begin{array}{l}\text { MESUROL } 500 \text { SC } \\
\text { /MeticarbMesurol }\end{array}$ & & Methiocarb & 972-2000-AG-SENASA \\
\hline Acaricidas & Acarex & Acarex & & & \\
\hline \multirow[t]{2}{*}{$\begin{array}{l}\text { Insecticida } \\
\text { /acaricida }\end{array}$} & Spider & Spider 1.8 EC & Abamectin & Abamectina & 889-99-AG-SENASA \\
\hline & Abosac & ABASAC $1.8 \mathrm{EC}$ & Abamectin & & 484-97-AG-SENASA \\
\hline Insecticida & Bamectin & Bamectin & Abamectin & Abamectina & $\begin{array}{l}\text { PQUA N }{ }^{\circ} \text { 965-SENASA / 396- } \\
\text { 97-AG-SENASA }\end{array}$ \\
\hline \multirow[t]{2}{*}{ Herbicida } & Gramoxone & Gramoxonesuper * & Paraquat & Paraquat & $\begin{array}{l}\text { (14 productos tienen registro de } \\
\text { SENASA) }\end{array}$ \\
\hline & Hielo sato & Glifosato & Glyphosate & Glifosato & $\begin{array}{l}\text { ( } 49 \text { productos tienen registro de } \\
\text { SENASA) }\end{array}$ \\
\hline Adherentes & Green Aderal & & Óxido nonyl fenol etileno & & \\
\hline \multirow[t]{2}{*}{ Foliares } & Orgavit & \multirow{2}{*}{\multicolumn{4}{|c|}{ (Quelato líquido de microelementos para aplicación radicular) }} \\
\hline & $\begin{array}{l}202020 \\
\text { (Fertiphos) } \\
121217 \\
\text { Bayfolan }\end{array}$ & & & & \\
\hline
\end{tabular}

Fuente: Trabajo de campo y consultas en SENASA (Servicio Nacional de Sanidad Agraria). Sistema Integrado de Gestión de Insumos Agropecuarios - SIGIA: http://200.60.104.77/SIGIAWeb/sigia_consulta_producto.html http://www.senasa.gob.pe/RepositorioAPS/0/0/JER/SUB_SEC_NOR/ANEXO\%201.PDF 
Sobre el paraquat, el principal cuestionamiento es que dadas las condiciones de producción predominantes en nuestro país no hay condiciones seguras para usar este plaguicida, particularmente por los pequeños agricultores. Además, no existe un antídoto. Los problemas han sido encontrados en trabajadores de los países en desarrollo quienes han sufrido daño a los pulmones, la piel, los ojos, la nariz y uñas de manos y pies. A la fecha 11 países han prohibido o restringido severamente su uso (Madeley, 2003).

En el caso de los herbicidas cuyo principio activo es el glyphosate, recientes estudios toxicológicos indican que los herbicidas que contienen este ingrediente activo pueden ser altamente tóxicos para los seres humanos y el medio ambiente. Se recomienda prohibir su uso por sus efectos agudos y crónicos sobre la salud humana, como también por la contaminación y pérdida de la biodiversidad que produce en el medio ambiente (RAP-AL, 2008).

En octubre del 2011 SENASA dispuso la suspensión temporal de los plaguicidas comerciales con ingredientes activos benomyl y paraquat $\left(\mathrm{RJ} \mathrm{N}^{\circ}\right.$ 307-2011-AG-SENASA), sin embargo, estas suspensiones fueron anuladas en enero del 2013 para proceder a una nueva evaluación toxicológica, ecotoxicológica y agronómica. (RJ Nº 0013-2012AG-SENASA). Actualmente (2013) los plaguicidas con los ingredientes activos benomyl y paraquat mantienen sus registros vigentes.

Otras investigaciones recientes en el Perú muestran que existe uso indiscriminado de pesticidas y ausencia de control sanitario en productos para el mercado interno y que los controles no son suficientemente rigurosos en comparación con los realizados para los alimentos destinados a la exportación (Delgado et al., 2018).

\section{- Los acopiadores}

Los acopiadores son actores de la cadena que congregan volúmenes de rocoto proveniente de los productores. Se ubican en espacios alrededor de las poblaciones y al borde de las carreteras principales de las zonas de producción (Figura 5). Cuentan con áreas para el acopio, selección y empaque del fruto en jabas. Son aproximadamente 30 acopiadores en la zona, permanecen durante todo el año y en las épocas de mayor cosecha se incrementa este número con acopiadores temporales. En Oxapampa y Villa Rica se encuentran los más importantes centros de acopio para la recepción del producto y su despacho hacia Lima.

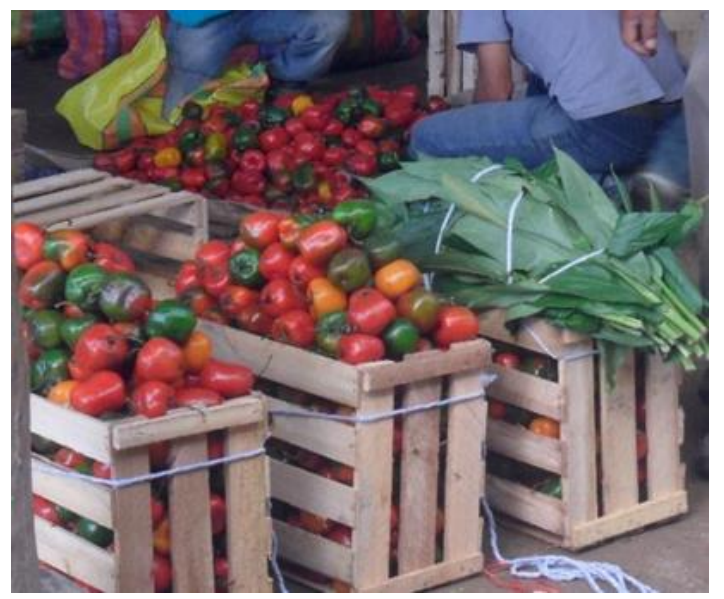

Figura 5. Selección y empacado de Rocoto. Oxapampa 2013.

Los acopiadores realizan la selección del rocoto según tamaño y en ese proceso aplican fungicidas al fruto antes de ser empacado. Realizan esta práctica para preservar el aspecto del fruto y neutralizar la acción de los hongos y facilitar la comercialización. Considerando que el rocoto cosechado en esta zona tiene altas probabilidades de que sea consumido en los dos o tres días siguientes, esta práctica es bastante riesgosa ya que los fungicidas son aplicados al momento del embarque y el periodo de carencia recomendado para estos plaguicidas es de 7 días. Esto es más preocupante ya que la principal forma de consumo del rocoto es en fresco, sea entero o molido.

\section{-Los Mayoristas}

Durante las visitas y el seguimiento efectuado a estos actores no se ha observado el uso de agroquímicos, pero actúan en favor de estas prácticas ya que propician su uso a través de los requerimientos de calidad que exigen en el momento de la compra del rocoto. Estos requerimientos han sido estandarizados por los mayoristas con el establecimiento, a la fecha del trabajo de campo, de 4 categorías de rocoto fresco. Estos corresponden a 
frutos de rocotos de diferentes tamaños, de forma, color rojo, con brillo y sin manchas. Estos fueron catalogados como rocoto: bola, primera, extra y super extra (Figura 6). Los precios mayoristas se establecen correlacionados a estas exigencias.

Estos requerimientos de calidad y precios establecidos por los mayoristas se trasladan a los agentes que se encuentran hacia atrás en la cadena, es decir, a los transportistas y acopiadores y de estos a los productores agrícolas de rocoto quienes para cumplir con estas exigencias y obtener mejores precios recurren al uso intensivo de agroquímicos. En correspondencia a estas exigencias, en la zona de Oxapampa, se producen los frutos de mayor tamaño en comparación a otras zonas productoras. En el año de estudio, el $60 \%$ de la producción de Oxapampa correspondió al tamaño extra y super- extra.

De esta forma los agentes mayoristas traducen los requerimientos de los minoristas, en especial de supermercados, restaurantes y empresas, que, al mismo tiempo, interpretan las exigencias de los consumidores.

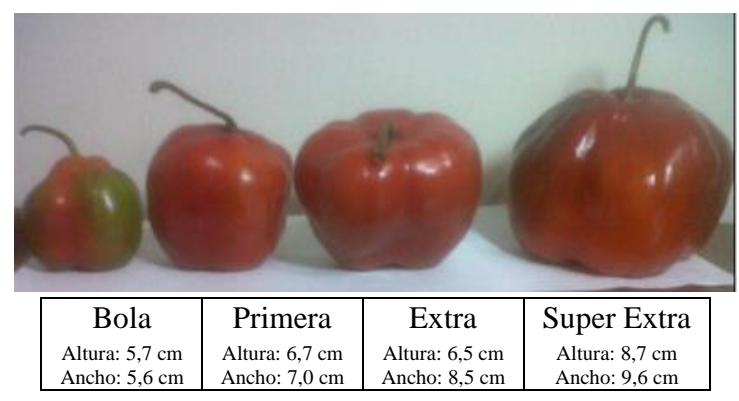

Figura 6. Rocoto por tamaño y denominaciones: Oxapampa 2013.

\section{-Los Minoristas.}

Se han identificado principalmente 2 grupos de minoristas en la cadena del rocoto. Los mercados minoristas de los diferentes distritos de Lima Metropolitana, que según cifras oficiales fueron más de 1200 mercados de abastos (año 2013). En volúmenes de venta de rocoto, estos minoristas son los más importantes y venden a las diferentes poblaciones sobre todo rocoto fresco y molido artesanalmente.
Otro agente minorista, que cada vez cobra más importancia, son los supermercados que ya eran 150 en el año 2013. Son los agentes de la cadena que tienen más importancia en la definición de los requerimientos y clasificación del rocoto, al interpretar los deseos de los consumidores y registrar las exigencias y tendencias del consumo.

\section{Valorización económica de la cadena productiva de rocoto}

El valor agregado de la cadena se estimó a partir del valor de los ingresos obtenidos por la comercialización del rocoto menos el costo del proveedor y el costo de operación en los que incurran los intermediarios. Estos gastos son: en transporte, financiamiento, y otros como pago de locales y re- selección.

A partir de esta información se midió el valor agregado generado en la cadena del rocoto que fue producido en Oxapampa y vendido en Lima Metropolitana. La Figura 7 muestra gráficamente las diferencias entre los costos totales y/o de adquisición, los costos operativos y los saldos netos correspondientes a cada eslabón en la cadena de rocoto.

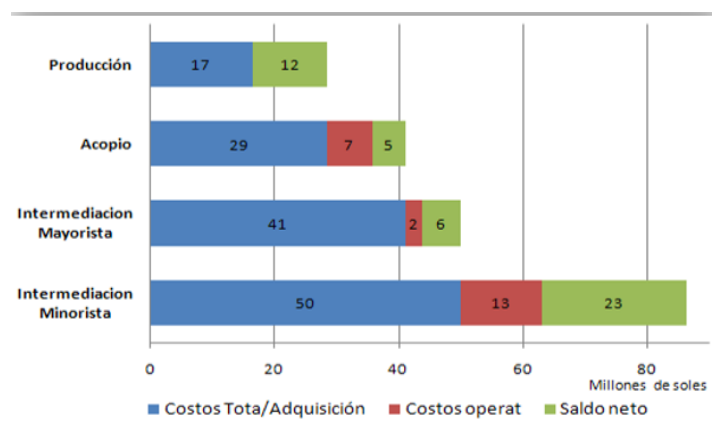

Figura 7. Resultado de la valoración económica de la cadena de valor de rocoto.

Las diferencias entre los precios pagados y los recibidos explican y definen la participación de cada eslabón en el total del valor agregado de la cadena. Todos los eslabones captan buena proporción del valor agregado, pero son los minoristas y los productores, los eslabones que captan la mayor proporción en el valor agregado total, el 50\% y el $26 \%$ respectivamente. En el caso del rocoto la variación porcentual de los precios mayoristas respecto a los precios minoristas fue mayor al $100 \%$. 
Ver Boletín: Semáforo de precios del 2013 (MINAGRI, 2013).

Los resultados explican la permanencia de los distintos agentes en esta cadena. Los productores continuarán produciendo con la misma tecnología e insistiendo en el uso de agroquímicos para lograr los tamaños extra y súper-extra que tienen la más alta cotización y una demanda segura en el mercado de Lima. De este modo los consumidores contribuyen a las malas prácticas de producción y comercialización.

\section{CONCLUSIÓN}

Entre Oxapampa, zona productora de rocoto y el mercado de Lima se configura una cadena de valor que se caracteriza por la fuerte presencia del mercado mayorista, los acopiadores y transportistas como agentes intermediarios entre los productores y los consumidores del mercado de Lima. La demanda por rocoto es creciente incentivada por los minoristas en especial de los supermercados. El consumidor tiene una marcada preferencia por rocotos de mayor tamaño, frescos y sin manchas. Estos requerimientos son transmitidos vía el mercado mayorista y los acopiadores a los productores quienes para lograr esta exigencia incrementan las áreas y recurren al uso intensivo de agroquímicos: fertilizantes y plaguicidas El uso de plaguicidas y el incentivo a su utilización no termina en la etapa productiva, sino que continúa en la etapa de mercadeo y el consumo. Es común el uso de plaguicidas recomendados para otros cultivos y con ingredientes activos prohibidos y de uso restringido en otros países (benomyl, paraquat y glyphosate) con los consiguientes riesgos para la salud, el medio ambiente y la biodiversidad. El análisis económico de la cadena de valor del rocoto muestra que, en promedio, los diferentes eslabones están obteniendo saldos netos positivos que hacen atractiva esta actividad productiva incentivando la producción con las características exigidas por la demanda y el consiguiente uso intensivo de agroquímicos.

Si no se promueven buenas prácticas en el uso de los plaguicidas se pone en riesgo la continuidad de esta importante actividad productiva por la intensidad del uso y los efectos en el suelo, el medio ambiente y la salud de productores y consumidores.
Se recomienda continuar investigando sobre los temas de contaminación en la producción agrícola y los alimentos con el fin de generar información para que los consumidores cuenten con casos reales que les permitan variar las actuales exigencias sobre los productos agrícolas porque afectan la salud y al medio ambiente.

\section{Conflictos de intereses}

Los autores firmantes del presente trabajo de investigación declaran no tener ningún potencial conflicto de interés personal o económico con otras personas $\mathrm{u}$ organizaciones que puedan influir indebidamente con el presente manuscrito.

\section{Contribuciones de los autores}

Preparación y ejecución: VGG. Desarrollo de la metodología: VGG. Concepción y diseño: VGG. Edición del artículo: VGG. Supervisión del estudio: VGG.

\section{LITERATURA CITADA}

- APEGA. [Sociedad Peruana de Gastronomía]. (2009). Ajíes peruanos. Sazón para el mundo. Lima, Perú. 122p.

- Astudillo, M., Solís, J., Silva, M., \& Maldonado, R. (2019). Cadenas agroalimentarias e innovación social: Perspectivas entre la competitividad y la sostenibilidad, Chilpancingo, México: Universidad Autónoma de Guerrero M; Seropédica, Brasil: Universidad Federal Rural do Rio de Janeiro. 135 pp. Disponible en: http://ri.uagro.mx/handle/uagro/1687

- Briz, J. (2011). La Cadena de Valor como instrumento de análisis del funcionamiento y riesgo del Sistema Agroalimentario. En La Cadena de Valor Agroalimentaria. Análisis internacional de casos reales, 67-95 pp, Coord. Julián Briz, Isabel de Felipe. España. Edit. Agrícola Española. 2011.

- Delgado, J., Alvarez, A., \& Yañez, J. (2018). Uso indiscriminado de pesticidas y ausencia de control sanitario para el mercado interno en el Perù. Pan American Journal of Public Health, 1 $-6$. 
- FAO- FAOSTAT. (2012). Estadísticas Mundiales de producción con relación a la alimentación y agricultura. Disponible en http://www.fao.org/faostat/es/\#data/QCL

- Madeley, J. (2003). Paraquat, el controvertido herbicida de Syngenta. Informe escrito para: Berne Declaration, Swedish Society for Nature Conservation, Pesticide Action Network UK, Pesticide Action Network Asia Pacific, Foro Emaús, RAP-AL. Consultado en: https://silo.tips/download/p-araquat-elcontrovertido-herbicida-de-syngenta-johnmadeley

- MINAGRI. [Ministerio de Agricultura y Riego]. 2013. Semáforo de precios Margen Bruto Minorista de Comercialización en Mercados de Lima Metropolitana. Oficina de Estudios Económicos y Estadísticos. Boletines 2013. Consultados en: https://www.midagri.gob.pe/portal/semafor o-precios/semaforo-2015/category/21semaforo-de-precios

- MINAGRI. [Ministerio de Agricultura y Riego]. (2013). SISAP (Sistema de Información de Abastecimiento y Precios). Disponible en http://sistemas.minag.gob.pe/sisap/portal/

- INEI. (2012). IV Censo nacional agropecuario. Sistema de consulta de resultados censales. Disponible en http://proyectos.inei.gob.pe/cenagro/tabulados/? $\mathrm{id}=$ CensosNacionales
- $\quad$ RALLT. [La Red por una América Latina Libre de Transgénicos]. (2015). ¿Qué sigue después de una prohibición del glifosato - más productos químicos tóxicos y cultivos transgénicos? Publicado por: African Centre For Biodiversity 21 p. Consultado en: http://www.rallt.org/PUBLICACIONES/Inform e\%20glifosato.pdf

- Sardón, E. (2015). Fortalecimiento de la cadena de valor para el desarrollo sostenible del rocoto (Capsicum pubescens) en la selva central del Perú. Tesis de Magister Scientiae, Universidad Nacional Agraria La Molina, Lima Perú.126 pp

- SENASA. [Servicio Nacional de Sanidad Agraria]. Sf. Normas nacionales sobre plaguicidas. Disponible en:

https://www.senasa.gob.pe/senasa/normasnacionales-sobre-plaguicidas/

- SENASA. [Servicio Nacional de Sanidad Agraria]. Sf. Sistema Integrado de Gestión de Insumos Agropecuarios - SIGIA. Disponible en: https://www.senasa.gob.pe/senasa/plaguicidasrestringidos-y-prohibidos-en-el-peru/

- SUNAT-ADUANAS. [Superintendencia Nacional de Aduanas y de Administración Tributaria]. Sf. Operatividad Aduanera. Descarga de Información. Disponible en: http://www.aduanet.gob.pe/aduanas/inform ae/aepartmen.htm. 